\title{
On realization of Siegel domains of the second kind as those of the third kind
}

\author{
By \\ Kazufumi NAKAJIMA
}

(Received Jan. 22, 1975)

\section{Introduction}

Let $D$ be a Siegel domain of the second kind associated with a convex cone $V$ and a $V$-hermitian form $F$. Realization of $D$ as a Siegel domain of the third kind was studied by Pyatetski-Shapiro [5], Wolf-Korányi [13] and by Satake [6] when $D$ is symmetric.

Kaneyuki [1] and Takeuchi [8] treated the case where $D$ is homogeneous. Their methods are based on the correspondence between " $j$-algebras" and "homogeneous Siegel domains of the second kind".

The purpose of the present paper is to prove that $D$ can be realized as a Siegel domain $\mathscr{D}$ of the third kind in such a way that the group $\operatorname{Aut}(D)$ acts on $\mathscr{D}$ as quasi-linear transformations. This is a generalization of a result of Takeuchi [8]. ${ }^{1)}$

In $\S 1$, we recall some results in [2] and [4] on the structute of the Lie algebra $\mathfrak{g}(D)$ of Aut $(D)$ and construct a symmetric domain $S$ which corresponds to a semi-simple part of $\mathfrak{g}(D)$. We also recall Tanaka's imbedding of the domain $D$ ([10], [3]).

In $\S 2$, we study a Cartan decomposition of $\mathfrak{g}(D)$ assuming that $D$ is symmetric. Many results in this section can be obtained also from Satake [7]. But our methods and proofs seem to be more direct and simpler.

1) Takeuchi [7] obtained this result for the identity component of Aut (D) when D is homogeneous. 
By using the results in previous sections, we shall study in $\S 3$ the structure of the cone $V$ and introduce a cone $V_{r}$ and a $V_{r}$-hermitian form $H$.

Finally in $\S 4$, by the same arguments as in Satake [6], we construct a Siegel domain $\mathscr{D}$ of the third kind, with $S$ as a base space, whose fiber is the Siegel domain of the second kind associated with $V_{r}$ and $H$. And making use of Tanaka's imbedding, we shall see that $D$ and $\mathscr{D}$ are holomorphically equivalent.

\section{$\S 1$. Summary of known results.}

1. 1. Let $R$ (resp. $W$ ) be a real (resp. complex) vector space of a finite dimension. Denote by $R_{c}$ the complexification of $R$. For every vector $z \in R_{c}$, we denote by $\operatorname{Re} z$ (resp. by $\operatorname{Im} z$ ) its real (resp. imaginary) part. ${ }^{2)}$

Let $D$ be a Siegel domain of the second kind in $R_{c}+W$, due to Pyatetski-Shapiro [5], associated with a convex cone $V$ in $R$ and a $V$-hermitian form $F$ on $W$, and let $g(D)$ be the Lie algebra of Aut $(D)$, the group of all holomorphic transformations of the domain $D$. Denote by $E$ (resp. by $I$ ) the element of $\mathfrak{g}(D)$ induced by the following one parameter group in $\operatorname{Aut}(D)$ (with parameter $t$ ):

$$
\begin{aligned}
& z+w \rightarrow e^{-2 t} z+e^{-\imath} w \quad\left(z \in R_{c}, w \in W\right) \\
& \text { (resp. } \left.z+w \rightarrow z+e^{\sqrt{-1} t} w\right) .
\end{aligned}
$$

Then from Kaup-Matsushima-Ochiai [2], the Lie algebra $\mathfrak{g}(D)$ has the following graded structure:

$$
\begin{aligned}
& \mathfrak{g}(D)=\mathfrak{g}^{-2}+\mathfrak{g}^{-1}+\mathfrak{g}^{0}+\mathfrak{g}^{1}+\mathfrak{g}^{2} \quad\left(\left[\mathfrak{g}^{\lambda}, \mathfrak{g}^{\mu}\right] \subset \mathfrak{g}^{\lambda+\mu}\right), \\
& \mathfrak{g}^{\lambda}=\{X \in \mathfrak{g}(D) ; \quad[E, X]=\lambda X\}, \\
& \mathfrak{r}=\mathfrak{r}^{-2}+\mathfrak{r}^{-1}+\mathfrak{r}^{0} \quad\left(\mathfrak{r}^{\lambda}=\mathfrak{r} \cap \mathfrak{g}^{\lambda}\right),
\end{aligned}
$$

where $r$ denotes the radical of $\mathfrak{g}(D)$.

We also know from [2] that both $E$ and $I$ are in the center of $\mathrm{g}^{0}$ and that $I$ has the following properties (cf. [3]):

$$
\text { ad } I=0 \text { on } \mathfrak{g}^{-2}+\mathfrak{g}^{0}+\mathfrak{g}^{2},
$$

2) In what follows, for a vector space or a Lie algebra $A$, we always mean by $A_{c}$ its complexification. 


$$
(\operatorname{ad} I)^{2}=-1 \text { on } \mathfrak{g}^{-1}+\mathfrak{g}^{1} \text {. }
$$

The space $\mathfrak{g}^{-2}$ (resp. $\mathfrak{g}^{-1}$ ) is identified with $R$ (resp. with $W$ ) in a natural manner. ${ }^{3)}$ Then the complex structure of $\mathrm{g}^{-1}$ is given by ad $I$, and the hermitian form $F$ and the domain $D$ are expressed as follows:

$$
\begin{aligned}
& F\left(w, w^{\prime}\right)=\frac{1}{4}\left(\left[[I, w], w^{\prime}\right]+\sqrt{-1}\left[w, w^{\prime}\right]\right), \\
& D=\left\{z+w \in \mathfrak{g}_{c}{ }^{-2}+\mathfrak{g}^{-1} ; \operatorname{Im} z-F(w, w) \in V\right\} .
\end{aligned}
$$

1.2. We now recall some results in [4]. There exists a semisimple graded subalgebra $\mathfrak{G}=\sum_{\lambda=-2}^{2} \mathfrak{G}^{\lambda}$ of $\mathfrak{g}(D)$ with the following properties :
i) $\mathfrak{g}^{1}=\mathfrak{g}^{1}$ and $\mathfrak{g}^{2}=\mathfrak{g}^{2}$.
ii) The adjoint representation of $\mathfrak{g}^{0}$ on $\mathfrak{g}^{1}+\mathfrak{g}^{2}$ is faithful.

Then $\mathfrak{g}^{-1}$ is a complex subspace of $\mathfrak{g}^{-1}$ and

$$
\begin{aligned}
& \mathfrak{g}^{-2}=\mathfrak{G}^{-2}+\mathfrak{r}^{-2} \quad \text { (direct sum) } \\
& \mathfrak{g}^{-1}=\mathfrak{g}^{-1}+\mathfrak{r}^{-1} \quad \text { (direct sum). }
\end{aligned}
$$

Since $\mathfrak{g}$ is semi-simple, there exists a unique $E_{s}$ in $\mathfrak{g}^{0}$ such that

$$
\mathfrak{G}^{\lambda}=\left\{X \in \mathfrak{g} ;\left[E_{\boldsymbol{s}}, X\right]=\lambda X\right\} .
$$

We set

$$
\begin{aligned}
& \mathfrak{r}_{s}^{-2}=\left\{X \in \mathfrak{r}^{-2} ;\left[E_{s}, X\right]=-X\right\}, \\
& \mathfrak{r}_{0}^{-2}=\left\{X \in \mathfrak{r}^{-2} ;[\mathfrak{g}, X]=0\right\}, \\
& \mathfrak{r}_{s}^{0}=\left\{X \in \mathfrak{r}^{0} ;\left[E_{s}, X\right]=X\right\}, \\
& \mathfrak{r}_{0}{ }^{0}=\left\{X \in \mathfrak{r}^{0} ;[\mathfrak{G}, X]=0\right\} .
\end{aligned}
$$

We then have

$$
\begin{aligned}
& \mathfrak{r}^{-2}=\mathfrak{r}_{s}{ }^{-2}+\mathfrak{r}_{0}{ }^{-2} \text { (direct sum) } \\
& \mathfrak{r}^{0}=\mathfrak{r}_{s}{ }^{0}+\mathfrak{r}_{0}{ }^{0} \text { (direct sum), }
\end{aligned}
$$

3) $\mathfrak{g}^{-2}$ (resp. $\mathbf{g}^{-1}$ ) consists of all elements of $\mathfrak{g}(D)$ induced by the following one parameter group (with parameter $t$ ):

$$
\begin{aligned}
& z+w \longrightarrow z+t a+w \quad(a \in R) \\
& (\text { resp. } z+w \longrightarrow z+2 \sqrt{-1} F(w, t c)+\sqrt{-1} F(t c, t c) \quad(c \in W)) .
\end{aligned}
$$




$$
\begin{aligned}
& \mathfrak{r}_{s}{ }^{-2}=\left[\mathfrak{r}^{-2}, \mathfrak{g}^{0}\right]=\left[\mathfrak{r}^{0}, \mathfrak{g}^{-2}\right] \supset\left[\mathfrak{r}^{-1}, \mathfrak{g}^{-1}\right] \\
& \mathfrak{r}_{s}^{0}=\left[\mathfrak{r}^{0}, \mathfrak{g}^{0}\right]=\left[\mathfrak{r}^{-2}, \mathfrak{g}^{2}\right] \supset\left[\mathfrak{r}^{-1}, \mathfrak{g}^{1}\right] .
\end{aligned}
$$

Moreover we know

$$
\left[E_{s}, \mathfrak{r}^{-1}\right]=0 .
$$

From (1.6), (1.7) and (1.8) we get

$$
\begin{aligned}
& {\left[\mathfrak{r}^{-1}, \mathfrak{r}^{-1}\right] \subset \mathfrak{r}_{0}^{-2},} \\
& {\left[\mathfrak{r}_{8}^{0}, \mathfrak{r}_{0}^{-2}+\mathfrak{r}^{-1}+\mathfrak{r}_{s}^{0}\right]=0} \\
& {\left[\mathfrak{r}_{8}^{0}, \mathfrak{r}_{s}^{-2}\right] \subset \mathfrak{r}_{0}{ }^{-2} .}
\end{aligned}
$$

For the algebra $\mathfrak{g}$, there exists a semi-simple subalgebra $\mathfrak{c}$ of $\mathfrak{g}^{0}$ such that

i) $[\mathfrak{G}, \mathfrak{c}]=0$.

ii) $\mathfrak{g}+\mathfrak{c}$ is a direct sum and is a semi-simple part of $\mathfrak{g}(D)$.

Note that the spaces $\mathfrak{r}_{s}{ }^{-2}, \mathfrak{r}_{0}{ }^{-2}, \mathfrak{r}_{s}{ }^{0}$ and $\mathfrak{r}_{0}{ }^{0}$ are stable by ad $X$ for $X \in \mathfrak{G}^{0}+\mathfrak{c}+\mathfrak{r}_{0}{ }^{0}$, because $\left[E_{s}, \mathfrak{g}^{0}+\mathfrak{c}+\mathfrak{r}_{0}{ }^{0}\right]=0$.

Let us denote by $\eta_{\mathfrak{s}}$ the projection of $\mathfrak{g}_{c}{ }^{-2}+\mathfrak{g}^{-1}$ onto $\mathfrak{G}_{c}{ }^{-2}+\mathfrak{g}^{-1}$ with respect to the decompositions (1.4). Then from (1.6), we get for any $v \in \mathrm{g}^{-2}$,

$$
\eta_{s}(v)=\lim _{t \rightarrow \infty} \frac{1}{e^{2 t}} A d\left(\exp \left(t E_{s}\right)\right) v
$$

Therefore if we put

$$
V_{s}=\eta_{s}(V), S=\eta_{s}(D),
$$

then $V_{s}$ is contained in $\bar{V}$, because $A d(\exp X) V=V$ for any $X \in \mathfrak{g}^{0.4)}$ Hence $V_{s}$ is an open convex cone is $\mathfrak{g}^{-2}$ containing no entire straight lines. Clearly the restriction $F_{s}$ of $F$ to $\mathfrak{g}^{-1} \times \mathfrak{g}^{-1}$ is a $V_{s}$-hermitian form. One of the main results in [4] is the following.

4) $\mathfrak{g}^{0}$ consists of all $A \in \mathfrak{g l}\left(R_{\mathrm{c}}+W\right)$ satisfying the followings: $A(R) \subset R, A(W) \subset W$, $\exp t A(V)=V$ and $A F\left(w, w^{\prime}\right)=F\left(A w, w^{\prime}\right)+F\left(w, A w^{\prime}\right)$. And under the iden. tification of $\mathfrak{g}^{-2}+\mathfrak{g}^{-1}$ with $R+W$, the equality; $A d(\exp A) X=\exp A(X)$ holds for any $X \in \mathbf{g}^{-2}+\mathfrak{g}^{-1}$. 
Theorem 1.1. $S$ is the symmetric Siegel domain of the second kink in $\mathfrak{S}_{c}{ }^{-2}+\mathfrak{g}^{-1}$ associated with $V_{s}$ and $F_{s}$, and the graded Lie algebra $\mathfrak{g}$ is identified with $\mathfrak{g}(S)$.

1.3. In this paragraph, we recall Tanaka's imbeddings. (For proofs of the following facts, see [10] and [3].)

Let $G$ be the identity component of Aut $(D)$ and let $g(D)_{c}$ be the complexification of $\mathfrak{g}(D)$. We denote by $G_{c}$ the adjoint group of $\mathfrak{g}(D)_{c}$. Since $G$ is centerless ([2]), we identify the Lie algebra $G_{c}$ with $\mathrm{g}(D)_{c}$ and $G$ with a subgroup of $G_{c}$. Define linear transformations $Q$ and $\bar{Q}$ of $\mathfrak{g}_{c}{ }^{-1}+\mathfrak{g}_{c}{ }^{1}$ by

$$
\begin{aligned}
& Q(X)=\frac{1}{2}(X-\sqrt{-1}[I, X]) \\
& \bar{Q}(X)=\frac{1}{2}(X+\sqrt{-1}[I, X]) \text { for } X \in \mathrm{g}_{c}{ }^{-1}+\mathfrak{g}_{c}{ }^{1} .
\end{aligned}
$$

We then have for $\lambda=-1,1$

$$
\begin{aligned}
& \mathfrak{g}_{c}{ }^{\lambda}=Q\left(\mathfrak{g}^{\lambda}\right)+\bar{Q}\left(\mathfrak{g}^{\lambda}\right) \quad(\text { direct sum }), \\
& Q\left(\mathfrak{g}^{\lambda}\right)=Q\left(\mathfrak{g}_{c}{ }^{\lambda}\right)=\left\{X \in \mathfrak{g}_{c}{ }^{\lambda} ;[I, X]=\sqrt{-1} X\right\} . \\
& \bar{Q}\left(\mathfrak{g}^{\lambda}\right)=\bar{Q}\left(\mathfrak{g}_{c}{ }^{\lambda}\right)=\left\{X \in \mathfrak{g}_{c}{ }^{\lambda} ;[I, X]=-\sqrt{-1} X\right\} .
\end{aligned}
$$

We set

$$
\mathfrak{b}=\bar{Q}\left(\mathfrak{g}^{-1}\right)+\mathfrak{g}_{c}{ }^{0}+\mathfrak{g}_{c}{ }^{1}+\mathfrak{g}_{c}{ }^{2} .
$$

Then $\mathfrak{b}$ is a complex subalgebra of $\mathfrak{g}(D)_{c}$ and $\operatorname{dim} \mathfrak{b}=\operatorname{dim} \mathfrak{g}(D)_{c}-\operatorname{dim} D$. Let $B$ be the closed subgroup of $G_{c}$ defined by

$$
B=\left\{g \in G_{c} ; g(\mathfrak{b})=\mathfrak{b}\right\} .
$$

The Lie algebra of $B$ coinsides with $\mathfrak{b}$ as is easily observed. We can now construct a map $h$ of $\mathfrak{g}_{c}{ }^{-2}+\mathfrak{g}^{-1}$ to $G_{c} / B$ as follows:

$$
h(z+w)=\pi \cdot \exp (z+Q(w)) \quad\left(z \in \mathfrak{g}_{c}{ }^{-2}, w \in \mathfrak{g}^{-1}\right),
$$

where $\pi$ denotes the projection of $G_{c}$ onto $G_{c} / B$. The map $h$ is holomorphic because $Q([I, w])=\sqrt{-1} Q(w)$. Moreover $h$ is an imbedding of $\mathfrak{g}_{c}{ }^{-2}+\mathfrak{g}^{-1}$ onto an open set of $G_{c} / B$ and satisfies the following

$$
h(g(p))=g \cdot h(p) \quad \text { for } \quad g \in G, p \in D .
$$


Remark 1. The mapping $h$ was first constructed by Tanaka [10] when the domain $D$ is homogeneous, and extended to general cases in [3].

\section{§ 2. Symmetric Siegel domains.}

2.1. Throughout this section, we assume that the Siegel domain $D$ is symmetric, which is equivalent to say that $\mathfrak{g}(D)$ is semi-simple.

Lemma 2. 1. Let $e \in V$. Then there exists a unique $e^{*}$ in $\mathfrak{g}^{2}$ such that $\left[e^{*}, e\right]=E$.

Proof. The uniqueness is obvious, since the mapping: $X \rightarrow[e, X]$ of $\mathrm{g}^{2}$ to $\mathrm{g}^{0}$ is injective (Vey [11]). We shall show the existence. Since $D$ is symmetric, the subalgebra $\mathfrak{g}^{\prime}=\mathfrak{g}^{-2}+\left[\mathfrak{g}^{-2}, \mathfrak{g}^{2}\right]+\mathfrak{g}^{2}$ is also semi-simple and $E$ is contained in $\left[\mathfrak{g}^{-2}, \mathfrak{g}^{2}\right]([4])$. We denote by $\rho$ the adjoint representaiton of $\left[\mathfrak{g}^{-2}, \mathfrak{g}^{2}\right]$ on $\mathfrak{g}^{-2}$. Let $\varphi_{V}$ be the characteristic function of $V$, which is a positive function defined on $V$ and satisfies the following equality (Vinberg [12]):

$$
\varphi_{V}(a x)=(\operatorname{det} a)^{-1} \varphi_{V}(x),
$$

where $a=\exp \rho(A)\left(A \in\left[\mathfrak{g}^{-2}, \mathfrak{g}^{2}\right]\right)$. Put $M(x)=\log \varphi_{V}(x)$. Since the killing form $\alpha^{\prime}$ of $\mathfrak{g}^{\prime}$ gives a duality between $\mathfrak{g}^{-2}$ and $\mathfrak{g}^{2}$, we can write in the Taylor series for $M(e+t x)$ as follows:

$$
M(e+t x)=M(e)-t \alpha^{\prime}(\tilde{e}, x)+0\left(t^{2}\right) .
$$

where $\tilde{e} \in \mathfrak{g}^{2}$. Then from (2.1), we obtain

$$
\alpha^{\prime}(\tilde{e},[A, e])=\operatorname{Tr} \rho(A) \quad \text { for } \quad A \in\left[\mathfrak{g}^{-2}, \mathfrak{g}^{2}\right] .
$$

Let $e^{*}=4 \tilde{e}$. Then $\alpha^{\prime}\left(\left[e^{*}, e\right], A\right)=-4 \operatorname{Tr} \rho(A)$. On the other hand from Tanaka [9], we get $\alpha^{\prime}(E, A)=2 \operatorname{Tr} \rho(E) \cdot \rho(A)=-4 \operatorname{Tr} \rho(A)$. Therefore $\alpha^{\prime}\left(E-\left[e^{*}, e\right], A\right)=0$ for any $A \in\left[\mathfrak{g}^{-2}, \mathfrak{g}^{2}\right]$ and hence $\alpha^{\prime}(E$ $\left.-\left[e^{*}, e\right], \mathfrak{g}^{\prime}\right)=0$. This implies $E=\left[e^{*}, e\right]$ q.e.d.

2. 2. We now investigate Cartan decompositions of the Lie algebra $\mathfrak{g}(D)$. Let $\mathfrak{g}(D)=\mathfrak{f}+\mathfrak{p}$ be the Cartan decomposition at the point $\sqrt{-1} e \in D(e \in V)$ and let $\sigma$ be the corresponding Cartan involution. 
Then $\mathfrak{f}$ is the isotropy subalgebra of $\mathfrak{g}(D)$ at $\sqrt{-1} e$. Therefore from $\cdots[2]$, we have

$$
\begin{aligned}
& \mathfrak{t}=\mathfrak{l}^{0}+\mathfrak{m}+\mathfrak{l} \quad \text { (direct sum), } \\
& \mathfrak{f}^{0}=\mathfrak{t} \cap \mathfrak{g}^{0}=\left\{A \in \mathfrak{g}^{0} ;[e, A]=0\right\}, \\
& \mathfrak{m}=\left\{X+\frac{1}{2}[e,[e, X]] ; X \in \mathfrak{g}^{2}\right\}, \\
& \mathfrak{n}=\left\{Y+[I,[e, Y]] ; Y \in \mathfrak{g}^{1}\right\} .
\end{aligned}
$$

We set

$$
\begin{aligned}
& \widetilde{\mathfrak{m}}=\left\{X-\frac{1}{2}[e,[e, X]] ; X \in \mathfrak{g}^{2}\right\}, \\
& \widetilde{\mathfrak{n}}=\left\{Y-[I,[e, Y]] ; Y \in \mathfrak{g}^{1}\right\} .
\end{aligned}
$$

Lemma 2.2. $\mathfrak{p} \supset \widetilde{\mathfrak{n}}+\widetilde{\mathfrak{m}}$.

Proof. Let $\alpha$ denote the killing form of $\mathfrak{g}(D)$. Then $\mathfrak{p}=\{X$ $\in \mathfrak{g}(D) ; \alpha(X, \mathfrak{f})=0\}$. Clearly $\alpha\left(\widetilde{\mathfrak{m}}+\widetilde{\mathfrak{n}}, \mathfrak{f}^{0}\right)=\alpha(\widetilde{\mathfrak{m}}, \mathfrak{n})=\alpha(\widetilde{\mathfrak{n}}, \mathfrak{m})=0$, because $\alpha\left(\mathfrak{g}^{\lambda}, \mathfrak{g}^{\mu}\right)=0$ for $\lambda+\mu \neq 0$. Let $X, X^{\prime} \in \mathfrak{g}^{2}$. Then

$$
\begin{aligned}
\alpha\left(X-\frac{1}{2}[e\right. & {\left.[e, X]], X^{\prime}+\frac{1}{2}\left[e,\left[e, X^{\prime}\right]\right]\right) } \\
& =\frac{1}{2} \alpha\left(X,\left[e,\left[e, X^{\prime}\right]\right]\right)-\frac{1}{2} \alpha\left(\left[e,[e, X], X^{\prime}\right)=0 .\right.
\end{aligned}
$$

Therefore we have $\alpha(\widetilde{\mathfrak{m}}, \mathfrak{m})=0$ and hence $\widetilde{\mathfrak{m}} \subset \mathfrak{p}$. By using (1.1) we can show $\alpha(\widetilde{\mathfrak{n}}, \mathfrak{n})=0$ similarly.

q.e.d.

Since $\sigma=1$ on $\mathfrak{f}$, we have for any $X \in \mathfrak{g}^{2}$

$$
X+\frac{1}{2}[e,[e, X]]=\sigma(X)+\frac{1}{2}[\sigma(e),[\sigma(e), \sigma(X)]] .
$$

On the other hand, $\sigma=-1$ on $\mathfrak{p}$. It follows from Lemma 2.2

$$
-X+\frac{1}{2}[e,[e, X]]=\sigma(X)-\frac{1}{2}[\sigma(e),[\sigma(e), \sigma(X)]] .
$$

Therefore we get

$$
\sigma(X)=\frac{1}{2}[e,[e, X]] \text { for } X \in \mathfrak{g}^{2} .
$$

Similarly we have

$$
\sigma(Y)=[I,[e, X]] \text { for } Y \in \mathfrak{g}^{1} .
$$

Let $e^{*}$ be as in Lemma 2.1. Then $\frac{1}{2}\left[e^{*},\left[e^{*}, X\right]\right] \in \mathrm{g}^{2}$ for any $X \in \mathfrak{g}^{-2}$. 
Hence by (2.4) we have

$$
\begin{aligned}
\sigma\left(\frac{1}{2}\left[e^{*},\left[e^{*}, X\right]\right]\right) & =\frac{1}{4}\left[e,\left[e,\left[e^{*},\left[e^{*}, X\right]\right]\right]\right] \\
& =\frac{1}{4}\left[e,\left[e^{*}, 2 X\right]\right] \\
& =X
\end{aligned}
$$

here we use the fact that $E$ is in the center of $\mathfrak{g}^{0}$. Since $\sigma^{2}=1$, we have

$$
\sigma(X)=\frac{1}{2}\left[e^{*},\left[c^{*}, X\right]\right] \quad \text { for } X \in \mathfrak{g}^{-2} .
$$

Similarly by using (1.1), we get

$$
\sigma(Y)=-\left[I,\left[e^{*}, Y\right]\right] \text { for } Y \in \mathfrak{g}^{-1} .
$$

By (2.4), (2.5), (2.6) and (2.7), we know that $\sigma\left(\mathfrak{g}^{\lambda}\right)=\mathfrak{g}^{-\lambda}$ for $\lambda$ $=-2,-1,1$ and 2. Hence $\sigma\left(\mathfrak{g}^{0}\right)=\mathfrak{g}^{0}$, because $\mathfrak{g}^{0}=\left[\mathfrak{g}^{-2}, \mathfrak{g}^{2}\right]+\left[\mathfrak{g}^{-1}, \mathfrak{g}^{1}\right]$ ([4]). Clearly $\sigma(e)=-e^{*}$ and $\sigma\left(e^{*}\right)=-e$. Therefore $\sigma(E)=-E$. We now assert $\left[e, \mathfrak{g}^{2}\right]=\left[e^{*}, \mathfrak{g}^{-2}\right]$. In fact $\mathfrak{g}^{2}=\left[e^{*},\left[e^{*}, \mathfrak{g}^{-2}\right]\right]$. So, $\left[e, \mathfrak{g}^{2}\right]$ $=\left[e^{*},\left[e,\left[e^{*}, \mathfrak{g}^{-2}\right]\right]\right]=\left[e^{*}, \mathfrak{g}^{-2}\right]$, proving our assertion. We set

$$
\mathfrak{p}^{0}=\left[e, \mathfrak{g}^{2}\right]=\left[e^{*}, \mathfrak{g}^{-2}\right] \text {. }
$$

Then $\mathfrak{f}^{0} \cap \mathfrak{p}^{0}=0$, because the mapping: $X \rightarrow[e,[e, X]]$ of $\mathfrak{g}^{2}$ to $\mathfrak{g}^{-2}$ is injective (cf. (2.2)). Moreover $\operatorname{dim} \mathfrak{g}^{0}=\operatorname{dim} \mathfrak{f}^{0}+\operatorname{dim} \mathfrak{g}^{-2}=\operatorname{dim} \mathfrak{f}^{0}+\operatorname{dim} \mathfrak{p}^{0}$. Hence we get $\mathfrak{g}^{0}=\mathfrak{f}^{0}+\mathfrak{p}^{0}$ (direct sum). Being invariant by $\sigma, \mathfrak{p}^{0}$ is containd in $\mathfrak{p}$. Thus we have proved.

Theorem 2.3. Let $D$ be a symmetric Siegel domain of the second kind and let $\mathfrak{g}(D)=\mathfrak{f}+\mathfrak{p}$ be the Cartan decomposition at the point $\sqrt{-1} e(e \in V)$. Then

$$
\begin{aligned}
& \mathfrak{f}=\mathfrak{f}^{0}+\mathfrak{m}+\mathfrak{l l} \quad \text { (direct sum) } \\
& \mathfrak{p}=\mathfrak{p}^{0}+\widetilde{\mathfrak{m}}+\widetilde{\mathrm{n}} \quad(\text { dircct } \text { sum }),
\end{aligned}
$$

where $\mathfrak{f}^{0}, \mathfrak{m}, \mathfrak{m}, \tilde{m}, \tilde{\pi}$ and $\mathfrak{p}^{0}$ are given by (2.2), (2.3) and (2.8).

2. 3. Let us denote by $\tilde{A}$ the holomorphic vector field on $D$ corresponding to $A \in \mathfrak{g}(D)$. Put $q=\sqrt{-1} e$. It is easy to see that the following equalities hold: 


$$
\begin{array}{lll}
\tilde{A}_{q}=\sqrt{ }-1[\widetilde{A, c}]_{q} & \text { for } & A \in \mathfrak{g}^{0}, \\
{[\overparen{I, Y}]_{q}=\sqrt{-1} \widetilde{Y}_{q}} & \text { for } & Y \in \mathfrak{g}^{-1} .
\end{array}
$$

Let $J$ be the complex structure of $D$. For $X \in \mathfrak{g}^{2}$, we have $\widetilde{X}_{q}-\widetilde{\sigma(X)_{q}}$ $=-2 \widetilde{\sigma(X)_{q}}$, because $X+\sigma(X) \in \mathfrak{f}$ and hence $\widetilde{X}_{q}+\widetilde{\sigma(X)_{q}}=0$. On the other hand by $(2.4)$ and $(2.9), \widetilde{[e, X}]_{q}=\sqrt{ }=1[\widetilde{e, X]}, e]_{q}=-2 \sqrt{-1} \overparen{\sigma(X)_{q}}$. Therefore we have

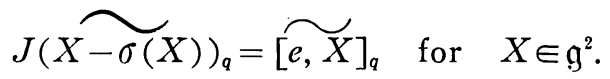

Similarly for $Y \in \mathrm{g}^{1}$, we have $\widetilde{Y}_{q}-\widetilde{\sigma(Y)_{q}}=-2 \widetilde{\sigma(Y)_{q}}$ and $\widetilde{I, Y]_{q}}-\widetilde{\sigma([I, Y])_{q}}$

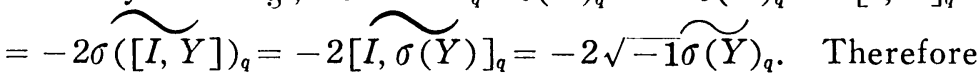

$$
J(\overbrace{Y-\sigma(Y))_{q}}=\overparen{[I, Y]_{q}}-\sigma([I, Y])_{q} \text { for } Y \in \mathfrak{g}^{1} .
$$

We set

$$
Z=\frac{1}{2}\left(I+e-e^{*}\right)=\frac{1}{2}(I+e+\sigma(e)) .
$$

Clearly $Z$ is contained in $\mathrm{f}$.

\section{Proposition 2. 4.}

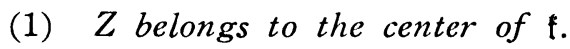

(2) Under the natural identification of $\mathfrak{p}$ with $T_{\sqrt{-1 e}}(D)$, the tangent space to $D$ at $\sqrt{-1} e$, the following equality holds:

$$
\text { ad } Z X=J X \text { for } X \in \mathfrak{p} \text {. }
$$

Proof. Since $\left[e, \mathfrak{f}^{0}\right]=0, \sigma\left(\left[e, \mathfrak{f}^{0}\right]\right)=\left[-e^{*}, \mathfrak{f}^{0}\right]=0 . \quad$ Let $X \in \mathfrak{g}^{2}$. Then by using (1.1), we obtain $[Z, X+\sigma(X)]=\frac{1}{2}([e, X]+[\sigma(e), \sigma(X)])$ $=\frac{1}{2}([e, X]+\sigma([e, X]))=0$, because $[e, X] \in \mathfrak{p}^{0}$ by Theorem 2.3. Therefore $[Z, \| 1]=0$. Next let $Y \in \mathfrak{g}^{1}$. Then from (1.1), (2.5) and (2. 7), $\quad[Z, Y+\sigma(Y)]=\frac{1}{2}([I, Y]+[I, \sigma(Y)]+[e, Y]+\sigma([e, Y]))$ $=\frac{1}{2}([I, Y]-[e, Y]+[e, Y]-[I, Y])=0$. Hence $[Z, \mathfrak{n}]=0$, proving (1).

By direct computations, we have

$$
[Z, X-\sigma(X)]=[e, X] \text { for } X \in \mathfrak{g}^{2} .
$$

(2.14) $[Z, Y-\sigma(Y)]=[I, Y-\sigma(Y)]$ 


$$
=[I, Y]-\sigma([I, Y]) \text { for } Y \in \mathfrak{g}^{1} .
$$

Now the statemet (2) follows immediately from (2.10), (2.11), (2.13) and (2.14).

q.e.d.

We set

$$
\begin{aligned}
& \mathfrak{p}_{-}=\left\{X \in \mathfrak{p}_{c} ;[Z, X]=-\sqrt{-1} X\right\}, \\
& \mathfrak{p}_{+}=\left\{X \in \mathfrak{p}_{c} ;[Z, X]=\sqrt{-1} X\right\}
\end{aligned}
$$

Then the following equalities hold:

$$
\begin{aligned}
& \mathfrak{p}_{c}=\mathfrak{p}_{+}+\mathfrak{p}_{-} \quad(\text { direct sum }), \\
& \mathfrak{p}_{+}=\left\{X-\sqrt{-1}[Z, X] ; X \in \mathfrak{p}_{c}\right\}, \\
& \mathfrak{p}_{-}=\left\{X+\sqrt{-1}[Z, X] ; X \in \mathfrak{p}_{c}\right\} .
\end{aligned}
$$

Proposition 2. 5. The following equlity holds:

$$
A d(\exp \sqrt{-1} e) \mathfrak{b}=\mathfrak{f}_{c}+\mathfrak{p}_{-},
$$

where $\mathfrak{b}$ is the subalgebra of $\mathfrak{g}(D)$ c given by (1.13).

Proof. Let $X \in \mathrm{g}_{\mathrm{c}}{ }^{2}$. By (2.13) we have $A d(\exp \sqrt{-1} e) X=X$ $+\sqrt{-1}[e, X]-\frac{1}{2}[e,[e, X]]=X-\sigma(X)+\sqrt{-1}$ ad $Z(X-\sigma(X))$. Therefore by (2.16), $A d(\exp \sqrt{-1} e) \mathfrak{g}_{c}{ }^{2} \subset \mathfrak{p}_{-} . \quad$ Next let $Y \in \mathfrak{g}_{c}{ }^{1}$. Then $A d(\exp \sqrt{-1} e) Y-\sigma(A d(\exp \sqrt{-1} e) Y)=Y+\sqrt{-1}[e, Y]-\sigma(Y)-\sqrt{-1}$ $\times[\sigma(e), \sigma(Y)]=Y-\sigma(Y)+\sqrt{-1}([I, Y]+[e, Y])=Y-\sigma(Y)+\sqrt{-1}$ $\times \operatorname{ad} Z(Y-\sigma(Y))$. This implies $A d(\exp \sqrt{-1} e) \mathfrak{g}_{c}{ }^{1} \subset \mathfrak{f}_{c}+\mathfrak{p}_{-}$. Let $A$ $=A_{1}+A_{2} \in \mathfrak{g}_{c}{ }^{0}$, where $A_{1} \in \mathfrak{f}_{c}{ }^{0}$ and $A_{2} \in \mathfrak{p}_{c}{ }^{0}$. It follows $A d(\exp \sqrt{-1} e) A$ $\equiv A_{2}+\sqrt{-1}\left[e, A_{2}\right]\left(\bmod \mathfrak{f}_{c}\right)$. And $A_{2}+\sqrt{-1}\left[e, A_{2}\right]-\sigma\left(A_{2}+\sqrt{-1}\left[e, A_{2}\right]\right)$ $=2 A_{2}+\sqrt{-1}\left[e, A_{2}\right]-\sqrt{-1}\left[e^{*}, A_{2}\right]=2\left(A_{2}+\sqrt{-1}\left[Z, A_{2}\right]\right) \in \mathfrak{p}_{-}$. Hence $A d(\exp \sqrt{-1} e) \mathfrak{g}_{c}{ }^{0} \subset \mathfrak{f}_{c}+\mathfrak{p}_{-}$. Finally for $Y \in \mathrm{g}_{c}{ }^{-1}, A d(\exp \sqrt{-1} e) \bar{Q}(Y)$ $=\bar{Q}(Y)$. Since $\sigma(I)=I$, it follows $\bar{Q}(Y)-\sigma \bar{Q}(Y)=\bar{Q}(Y-\sigma(Y))$. Hence by $(2.14)$, we get ad $Z(\bar{Q}(Y)-\sigma(\bar{Q}(Y)))=$ ad $I(\bar{Q}(Y-\sigma(Y))$ $=-\sqrt{-1}(\bar{Q}(Y)-\sigma \bar{Q}(Y))$. Therefore $\bar{Q}(Y)-\sigma(\bar{Q}(Y)) \in \mathfrak{p}_{-}$and hence $\bar{Q}\left(\mathfrak{g}_{c}{ }^{-1}\right) \subset \mathfrak{f}_{c}+\mathfrak{p}_{-} . \quad$ Thus we have proved $A d(\exp \sqrt{-1} e) \mathfrak{b} \subset \mathfrak{f}_{c}+\mathfrak{p}_{-}$. Since $\operatorname{dim}\left(\mathfrak{f}_{c}+\mathfrak{p}_{-}\right)=\operatorname{dim} \mathfrak{g}(D)_{c}-\operatorname{dim} D=\operatorname{dim} \mathfrak{l}, \quad$ we can conclude $A d(\exp \sqrt{-1} c) \mathfrak{b}=\mathfrak{f}_{\mathrm{r}}+\mathfrak{p}$.. q.e.d. 


\section{§. The structure of the cone $V$.}

3. 1. We return to general cases. Let $\eta_{r}$ denote the projection of $\mathfrak{g}^{-2}$ onto $\mathfrak{r}_{0}^{-2}$ with respect to the decomposition $\mathfrak{g}^{-2}=\mathfrak{g}^{-2}+\mathfrak{r}_{0}{ }^{-2}+\mathfrak{r}_{\mathfrak{s}}{ }^{-2}$. Put

$$
V_{r}=\eta_{r}(V) .
$$

By (1.6) we get for any $v \in \mathfrak{g}^{-2}$

$$
\eta_{r}(v)=\lim _{t \rightarrow \infty} A d\left(\exp t E_{\delta}\right) v .
$$

Therefore $V_{r} \subset \bar{V}$. From this fact, it follows that $V_{r}$ is an open convex cone in $x_{0}{ }^{-2}$ containing no entire straight lines. It is clear from (1.2) and (1.9) that

$$
F(w, w)=\frac{1}{4}[[I, w], w] \in \bar{V}_{r} \quad \text { for } \quad w \in \mathfrak{r}^{-1} .
$$

Let $v=e+a+b \in \mathfrak{g}^{-2}$, where $e \in V_{s}, a \in \mathfrak{x}_{0}^{-2}$ and $b \in \mathrm{r}_{s}{ }^{-2}$. Since the domain $S$, constructed in $\S 1$, is symmetric, there exists $e^{*}$ in $\mathfrak{g}^{2}\left(=\mathfrak{g}^{2}\right)$ such that $E_{s}=\left[e^{*}, e\right]$ by Lemma 2.1. We then have by (1.6), (1.7) and (1.10)

$$
\begin{aligned}
& A d\left(\exp \left[e^{*}, b\right]\right) e=e-b+\frac{1}{2}\left[\left[b, e^{*}\right], b\right], \\
& A d\left(\exp \left[e^{*}, b\right]\right) v=e+a-\frac{1}{2}\left[\left[b, e^{*}\right], b\right] .
\end{aligned}
$$

Since $V_{s}$ is contained in $\bar{V}$, we get from (3.2)

$$
\frac{1}{2}\left[\left[b, e^{*}\right], b\right] \in \bar{V}_{r} \quad \text { for any } b \in \mathrm{r}_{s}{ }^{-2} .
$$

And by (3.3)

$$
a-\frac{1}{2}\left[\left[b, e^{*}\right], b\right] \in V_{r} \quad \text { if } \quad v \in V .
$$

Let $\eta=\eta_{s}+\eta_{r}$, i.e., the projection of $\mathfrak{g}^{-2}$ to $\mathfrak{g}^{-2}+\mathrm{r}_{0}{ }^{-2}$. Since $x+y$ $\in V$ if $x \in V$ and $y \in \bar{V}$, we know from (3.2) and (3.4) that $\eta(V)$ is contained in $V$.

Lemma 3. 1. $V_{s}+V_{r}=\eta(V)$.

Proof. Clearly $V_{s}+V_{r} \supset \eta(V)$. Conversely let $e \in V_{s}$ and $a \in V_{r}$. Then $e+a \in \bar{V}$. Therefore $e+a \in \eta(\bar{V}) \subset \overline{\eta(V)}$. Hence $V_{s}+V_{r} \subset \overline{\eta(V)}$. Therefore $V_{s}+V_{r}$ is the interior of $\overline{\eta(V)}$. This implies $V_{s}+V_{r}$ 
$=\eta(V)$.

q.e.d.

Proposition 3. 2. Let $v=e+a+b \in \mathfrak{g}^{-2}$, where $e \in \mathfrak{g}^{-2}, a \in \mathfrak{r}_{0}{ }^{-2}$ and $b \in \mathrm{r}_{s}^{-2}$. Then $v \in V$ is and only if $e \in V_{s}$ and $a-\frac{1}{2}\left[\left[b, e^{*}\right], b\right]$ $\in V_{r}$.

Proof. The "if" part is already proved (cf. (3.5)). Suppose that $e \in V_{s}$ and $a-\frac{1}{2}\left[\left[b, e^{*}\right], b\right] \in V_{r}$. Then from Lemma 3. 1, $u=e+a$ $-\frac{1}{2}\left[\left[b, e^{*}\right], b\right] \in \eta(V) \subset V$. Since $a d\left(\exp \left(-\left[e^{*}, b\right]\right)\right) u=v$, we get $v \in V$.

q.e.d.

If we set

$$
D_{o}=\eta_{s}^{-1}(\sqrt{-1} e) \quad\left(e \in V_{s}\right),
$$

Then we get immediately from proposition 3.2

\section{Corollary 3. 3 .}

$$
\begin{aligned}
D_{o}=\left\{u+v+w+\sqrt{-1} e ; u \in\left(\mathrm{r}_{0}^{-2}\right)_{c}, v \in\left(\mathrm{r}_{s}^{-2}\right)_{c}, w \in \mathfrak{r}^{-1}\right. \\
\left.\operatorname{Im} u-\frac{1}{4}[[I, w], w]-\frac{1}{2}\left[\left[\operatorname{Im} v, e^{*}\right], \operatorname{Im} v\right] \in V_{r}\right\} .
\end{aligned}
$$

3. 2. By (1.7), ade (resp. ad $e^{*}$ ) gives a linear mapping of $\mathrm{r}_{s}{ }^{0}$ (resp. $\mathfrak{r}_{8}{ }^{-2}$ ) to $\mathfrak{r}_{s}{ }^{-2}$ (resp. to $\mathfrak{r}_{8}{ }^{0}$ ). Let $X \in \mathfrak{r}_{8}{ }^{-2}$ and $Y \in \mathfrak{r}_{8}{ }^{0}$. By (1.6), $\left[e,\left[e^{*}, X\right]\right]=\left[\left[e, e^{*}\right], X\right]=-\left[E_{s}, X\right]=X$ and $\left[e^{*},[e, Y]\right]$ $=\left[\left[e^{*}, e\right], Y\right]=\left[E_{s}, Y\right]=Y$. Thereby

$$
\begin{aligned}
& \operatorname{ad} e \cdot a d e^{*}=1 \quad \text { on } \quad \mathrm{r}_{s}{ }^{-2}, \\
& \operatorname{ad} e^{*} \cdot a d e=1 \quad \text { on } \mathrm{r}_{s}{ }^{0} .
\end{aligned}
$$

In particular, ad $e^{*}$ (resp. $a d c$ ) gives an isomorphism of $\mathrm{r}_{s}^{-2}$ (resp. of $\mathfrak{r}_{s}{ }^{0}$ ) onto $\mathfrak{r}_{s}{ }^{0}$ (resp. onto $\boldsymbol{r}_{s}{ }^{-2}$ ).

\section{Lemma 3. 4.}

(1) Let $b \in \mathrm{r}_{s}^{-2}$. Then $\left[\left[b, e^{*}\right], b\right] \in \bar{V}_{r}$ and $\left[\left[b, e^{*}\right], b\right]=0$ implies $b=0$.

(2) Let $c \in \mathrm{r}_{s}{ }^{0}$. Then $[[e, c], c] \in \bar{V}_{\tau}$ and $[[e, c], c]=0$ implies $c=0$. 
Proof. The fact $\left[\left[b, e^{*}\right], b\right] \in \bar{V}$, is already proved (cf. (3.4)). Suppose $\left[\left[b, e^{*}\right], b\right]=0$. Then by $(3.2), e-b$ is contained in $\bar{V}$. Since $\lim _{t \rightarrow \infty} e^{t} A d\left(\exp t E_{s}\right)(e-b)=-b$, we get $-b \in \bar{V}$. Similarly we have $b \in \bar{V}$, because $\left[\left[-b, e^{*}\right],-b\right]=0$. Now $b=0$ follows immediately from the fact that $\vec{V}$ contains no entire straight lines. Hence we have proved (1). We can write $c=\left[e^{*}, b^{\prime}\right], b^{\prime} \in r_{s}{ }^{-2}$. Then $[[e, c], c]=\left[\left[e,\left[e^{*}, b^{\prime}\right]\right],\left[e^{*}, b^{\prime}\right]\right]=\left[b^{\prime},\left[e^{*}, b^{\prime}\right]\right]=\left[\left[b^{\prime}, e^{*}\right], b^{\prime}\right]$. Therefore the assertion (2) follows from (3.7) and (1). q.e.d.

\section{Now we set}

$$
\begin{aligned}
& U=\mathrm{r}_{0}{ }^{-2}, \\
& \mathcal{W}=\mathrm{r}_{s}^{-2}+\mathrm{r}^{-1}+\mathrm{r}_{s}^{0}, \\
& j_{0}=\operatorname{ad}\left(I+c-e^{*}\right) .
\end{aligned}
$$

It follows from (1.1) and (3.7) that $j_{0}^{2}=-1$ on $\mathscr{W}$. Hence we can write

$$
\begin{aligned}
& W_{c}=W_{+}+W_{-} \quad \text { (direct sum), } \\
& W_{+}=\left\{w \in W_{c} ; j_{o} w=\sqrt{-1} w\right\}, \\
& W_{-}=\left\{w \in W_{c} ; j_{o} w=-\sqrt{-1} w\right\}, \\
& \bar{W}_{+}=W_{-}
\end{aligned}
$$

Clearly the following equalities hold:

$$
\left[W_{+}, W_{+}\right]=\left[W_{-}, W_{-}\right]=0
$$

Define a $U_{c}$-valued skew-symmetric biliear form $\mathcal{A}$ on $\mathcal{W}_{\mathrm{c}}$ by

$$
\mathscr{A}\left(w, w^{\prime}\right)=\frac{1}{4}\left[w, w^{\prime}\right] \quad\left(w, w^{\prime} \in W_{c}\right) .
$$

Proposition 3. 5. Let $H\left(w, w^{\prime}\right)=2 \sqrt{-1} \mathcal{A}\left(w, \bar{w}^{\prime}\right)$ for $w$, w $w^{\prime}$ $\in W_{+}$. Then $H$ is a $V_{r^{-}}$hermitian form on $\mathcal{W}_{+}$.

Proof. Each element $w$ of $W_{+}$can be written as $w=w_{1}+\sqrt{-1}$ $\times\left[e^{*}, w_{1}\right]+w_{2}-\sqrt{-1}\left[e, w_{2}\right]+Q\left(w_{3}\right)$, where $w_{1} \in \mathfrak{r}_{s}{ }^{-2}, w_{2} \in \mathfrak{r}_{s}^{0}$ and $w_{3}$ $\in \mathrm{r}^{-1}$. Then by using (1.10) and (3.11), we get 


$$
H(w, w)=\left[\left[w_{1}, e^{*}\right] ; w_{1}\right]+\left[\left[e, w_{2}\right], w_{2}\right]+\frac{1}{4}\left[\left[I, w_{3}\right], w_{3}\right]
$$

Hence by (3.1) and Lemma 3.4, we know that $H(w, w) \in \bar{V}_{r}$ and that $H(w, w)=0$ means $w_{1}=w_{2}=w_{3}=0$.

q.e.d.

\section{§ 4. Realization of $D$ as a Siegel domain of the third kind.}

4. 1. Let $S$ be the symmetric domain constructed in $\S 1$ and let $\mathfrak{g}(S)=\mathfrak{k}+\mathfrak{p}$ be the Cartan decomposition at $\sqrt{-1} e\left(e \in V_{s}\right)$. There exists a unique $I_{s}$ in $\mathfrak{g}^{0}$ such that $a d I_{s}=a d I$ on $\mathfrak{g}(=\mathfrak{g}(S))$. Then by Proposition 2.4, $Z=\frac{1}{2}\left(I_{s}+e-e^{*}\right)$ is in the center of $\mathfrak{l}$ and $a d Z$ gives a complex structure on $\mathfrak{p}$ which coincides with one of the domain $S$ under the natural identification of $\mathfrak{p}$ with the tangent space to $S$ at $\sqrt{-1} c$. Let $\mathfrak{p}_{+}$and $p_{-}$be the subspace of $p^{\prime}$ given by (2.15) for the domain $S$. Note that

$$
\begin{aligned}
& {\left[\mathfrak{p}_{+}, \mathfrak{p}_{+}\right]=\left[\mathfrak{p}_{-}, \mathfrak{p}_{-}\right]=0,} \\
& {\left[\mathfrak{p}_{+}, \mathfrak{p}_{-}\right] \subset \mathfrak{f}_{c},} \\
& \mathfrak{p}_{+}=\overline{\mathfrak{p}}_{-}, \\
& {\left[\mathfrak{f}_{c}, \mathfrak{p}_{ \pm}\right] \subset \mathfrak{p}_{ \pm}}
\end{aligned}
$$

Let $G^{s}, G_{c}^{s}, K, K_{c}, P_{+}$and $P_{-}$be the connected subgroups of $G_{c}$ corresponding to the subalgebras $\mathfrak{g} ; \mathfrak{g}_{c}, \mathfrak{f}, \mathfrak{f}_{c}, \mathfrak{p}_{+}$and $\mathfrak{p}_{-}$. Then $P_{ \pm}, K_{c}$ and $K_{c} P_{-}$are closed complex subgroups of $G_{c}{ }^{8}$. Moreover exp: $\mathfrak{p}_{ \pm} \rightarrow P_{ \pm}$ are holomorphic diffeomorphisms. It is also well known that the mapping defined by

$$
P_{+} \times K_{c} \times P_{-} \rightrightarrows(a, b, c) \rightarrow a b c \in G_{c}^{s}
$$

is a holomorphic diffeomorphism onto an open set of $G_{c}{ }_{c}$. and that $G^{s}$ $\subset P_{+} K_{c} P_{-}$. Therefore for each point $g K$ in $S=G^{s} / K$, there corresponds a unique $z$ in $\mathfrak{p}_{+}$such that exp $z=$ the $P_{+}$-part of $g$, and the assignment: $g K \rightarrow z$ gives a holomorphic imbedding of $S$ onto a bounded domain $\mathscr{S}$ in $\mathfrak{p}_{+}$. This is called the Harish-Chandra imbedding.

Let $z, z^{\prime} \in \mathscr{S}$. Then we can write $\exp z=g \cdot k \cdot p$, $\exp z^{\prime}=g^{\prime} \cdot k^{\prime} \cdot p^{\prime}$, where $g, g^{\prime} \in G^{s}, k, k^{\prime} \in K_{c}$ and $p, p^{\prime} \in P_{-}$. It follows that $\left(\exp \bar{z}^{\prime}\right)^{-1}$ $\cdot \exp z=\bar{p}^{\prime-1} \cdot \bar{k}^{\prime-1} \cdot g^{\prime-1} \cdot g \cdot k \cdot p$. Since $\dot{g}^{\prime-1} \cdot g \in P_{+} K_{c} P_{-} ;\left(\exp \bar{z}^{\prime}\right)^{-1} \cdot \exp z$ $\in P_{+} K_{c} P_{-}$. Thus we can define a mapping $\mathcal{K}\left(z, z^{\prime}\right): \mathscr{S} \times \mathscr{S} \rightarrow K_{c}$ by 


$$
\mathcal{K}\left(z, z^{\prime}\right)^{-1}=\text { the } K_{\mathrm{c}} \text {-part of }\left(\exp \bar{z}^{\prime}\right)^{-1} \cdot \exp z .
$$

It is easy to check the following equality (Satake [6]):

$$
\mathcal{K}\left(z^{\prime}, z\right)=\overline{\mathcal{K}\left(z, z^{\prime}\right)^{-1}}
$$

We also define a mapping $g(g, z): G^{s} \times \mathscr{S} \rightarrow K_{c}$ by

$$
g(g, z)=\text { the } K_{c} \text {-part of } g \cdot \exp z \text {. }
$$

The group $G^{s}$ acts on $\mathscr{S}$ in obvious manner. Then $g \cdot \exp z \equiv \exp g(z)$ $\cdot g(g, z)\left(\bmod P_{-}\right)$. By a simple calculation we have (Satake [6])

$$
\mathcal{K}\left(g(z), g\left(z^{\prime}\right)\right)=g(g, z) \cdot \mathcal{K}\left(z, z^{\prime}\right) \cdot \bar{g}(g, z)^{-1} .
$$

If we put $\mathcal{K}(z)=\mathcal{K}(z, z)$, then by (4.2) and (4.3) we know

$$
\begin{aligned}
& \mathcal{K}(z)=\bar{K}(z)^{-1} \quad(z \in \mathscr{S}), \\
& \mathcal{K}(g(0))=g(g, 0) \cdot \bar{g}(g, 0)^{-1} \quad\left(g \in G^{s}\right) .
\end{aligned}
$$

4. 2. Let $\mathcal{U}_{c}, W_{+}$and $\mathscr{W}_{-}$be as in $\S 3$. Clearly $\left[\mathfrak{s}_{c}, \mathcal{U}_{c}\right]=0$ and $\left[\mathfrak{g}_{c}, W_{c}\right] \subset W_{c}$ by $(1.6),(1.7)$ and (3.8). Since $j_{0} X=a d(I+e$ $\left.-e^{*}\right) X=a d\left(I_{s}+e-e^{*}\right) X$ for $X \in \mathfrak{g}$, we easily have the followings:

$$
\begin{aligned}
& {\left[p_{+}, W_{+}\right]=\left[p_{-}, W_{-}\right]=0,} \\
& {\left[p_{+}, W_{-}\right] \subset W_{+},} \\
& {\left[p_{-}, W_{+}\right] \subset W_{-},} \\
& {\left[\mathfrak{f}_{c}, W_{ \pm}\right] \subset W_{+} .}
\end{aligned}
$$

In what follows, we simply write the actions of $g \in G_{c}{ }^{s}$ and $z \in \mathfrak{g}_{c}$ on $W_{c}$ as $g w$ and $z w\left(w \in W_{c}\right)$. Since $\left[\mathfrak{B}_{c}, U_{c}\right]=0$, following equalities hold:

(4.6) $\mathcal{A}\left(g w, g w^{\prime}\right)=\mathcal{A}\left(w, w^{\prime}\right)$.

$$
\mathcal{A}\left(z w, w^{\prime}\right)+\mathcal{A}\left(w, z w^{\prime}\right)=0 \quad\left(g \in G_{c}{ }^{s}, z \in \mathfrak{g}_{c}, w, w^{\prime} \in W_{c}\right),
$$

where $\mathcal{A}$ is the skew-symmetric bilinear form on $\mathscr{W}_{c}$ defined by (3.12). We now define for each $z \in \mathscr{S}$ a $U_{c}$-valued form $L_{z}\left(w, w^{\prime}\right)$ on $\mathscr{W}_{+}$by

$$
L_{z}\left(w, w^{\prime}\right)=2 \sqrt{-1} \mathcal{A}\left(\overline{\mathcal{K}(z)} w, \bar{w}^{\prime}\right) \quad\left(w, w^{\prime} \in W_{+}\right) .
$$

Then by (4.4) and (4..6), $L_{z}\left(w, w^{\prime}\right)$ is hermitian. 
Lemma 4. 1 (cf. [6]).

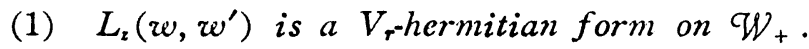

(2) $L_{z}\left(w, z \bar{w}^{\prime}\right)$ is a symmetric bilinear form on $\mathcal{W}_{+}$.

Proof. We can take an element $g \in G^{\prime}$ such that $g(0)=z$. Now (1) follows immediately from (4.4), (4.6) and from Proposition 3.5. To prove (2), we first show that the following equalities hold:

$$
\begin{aligned}
& (1-z \bar{z}) w_{+}=\mathcal{K}(z) w_{+} \text {for } w_{+} \in W_{+}, \\
& (1-\bar{z} z) w_{-}=\mathcal{K}(z)^{-1} w_{-} \text {for } w_{-} \in W_{-} .
\end{aligned}
$$

In fact, there exist $z_{1}, z_{2} \in \mathfrak{p}_{+}$such that $(\exp \bar{z})^{-1} \cdot \exp z=\exp z_{1} \cdot \mathcal{K}(z)^{-1}$ $\cdot \exp \bar{z}_{2}$. Hence by (4.5),

$$
\begin{aligned}
w_{-}+z w_{-}-\bar{z} z w_{-} & =(\exp \bar{z})^{-1} \cdot(\exp z) w_{-} \\
& =\exp z_{1} \cdot \mathcal{K}(z)^{-1} w_{-} \\
& =\mathcal{K}(z)^{-1} w_{-}+z_{1} \mathcal{K}(z)^{-1} w_{-} .
\end{aligned}
$$

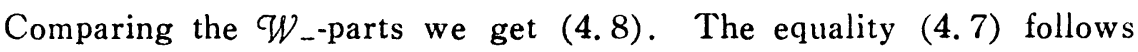
from (4.4) and (4.8). From (4.7), $\bar{z}(1-z \bar{z})=\bar{z} \mathcal{K}(z)$ on $\mathcal{W}_{+}$. And from (4.8), $(1-\bar{z} z) \bar{z}=\mathcal{K}(z)^{-1} \cdot \bar{z}$ on $\mathcal{W}_{+}$. Therefore $\bar{z} \cdot \mathcal{K}(z)=\mathcal{K}(z)^{-1} \cdot \bar{z}$ and hence $\mathcal{K}(z) \cdot \bar{z}=\bar{z} \cdot \overline{\mathcal{K}(z)}$ on $\mathcal{W}_{+}$. It follows

$$
\begin{aligned}
L_{z}\left(w, z \bar{w}^{\prime}\right) & =2 \sqrt{-1} \not{A}\left(\overline{\mathcal{K}(z)} w, \bar{z} w^{\prime}\right) \\
& =2 \sqrt{-1} \mathcal{A}\left(w, \mathcal{K}(z) \bar{z} w^{\prime}\right) \\
& =2 \sqrt{-1} \mathcal{A}\left(w, \bar{z} \cdot \overline{\mathcal{K}(z)} w^{\prime}\right) \\
& =-2 \sqrt{-1} \mathcal{A}\left(\bar{z} w, \overline{\mathcal{K}(z)} w^{\prime}\right) \\
& =2 \sqrt{-1} \mathcal{A}\left(\overline{\mathcal{K}(z)} w^{\prime}, \bar{z} w\right) \\
& =L_{z}\left(w^{\prime}, z \bar{w}\right) .
\end{aligned}
$$$$
\text { q.e.d. }
$$

We now set for $z \in \mathscr{S}$,

$$
\left.\mathcal{L}_{z}\left(w, w^{\prime}\right)=L_{z}\left(w, w^{\prime}\right)+L_{z}\left(w, z \bar{w}^{\prime}\right) \quad\left(w, w^{\prime} \in \mathscr{Q}\right)_{+}\right) .
$$

Then $\mathcal{L}_{z}$ is a non-degenerate semi-hermitian form on $\mathcal{W}_{+}$in the sence of Pyatetski-Shapiro [5]. Indeed, suppose that there exists $w_{o} \in W_{+}$ 
such that $\mathcal{L}_{z}\left(w, w_{0}\right)=0$ for any $w \in \mathcal{W}_{+}$. Then $w_{0}+z \bar{w}_{0}=0$ and hence $\bar{z} w_{0}+\bar{z} z \bar{w}_{0}=0$. It follows that $(1-\bar{z} z) \bar{w}_{0}=\bar{w}_{0}-\bar{z} z \bar{w}_{0}=\overline{\left(w_{0}+z \bar{w}_{0}\right)}$ $-\left(\bar{z} w_{0}+\bar{z} z \bar{w}_{0}\right)=0$. Since $1-\bar{z} z$ is non-singular (cf. Proof of Lemma 4. 1), we get $w_{0}=0$. Therefore $\mathcal{L}_{z}\left(w, w^{\prime}\right)$ is non-singular. Thereby we can define a Siegel domain $\mathscr{D}$ of the third kind by

$$
\mathscr{D}=\left\{(u, w, z) \in U_{c} \times \mathscr{W}_{+} \times \mathscr{S} ; \operatorname{Im} u-\operatorname{Re} \mathcal{L}_{z}(w, w) \in V_{r}\right\} .
$$

Let $\xi$ denote the natural projection of $\mathscr{D}$ onto $\mathscr{S}$ and let $\mathscr{D}_{o}=\xi^{-1}(0)$. Since $\mathcal{L}_{0}\left(w, w^{\prime}\right)=H\left(w, w^{\prime}\right)$, we get

Proposition 4. 2. The fiber $\mathscr{D}_{0}$ is the Siegel domain of the second kind associated with the cone $V_{r}$ and the $V_{r}$-hermitian form $H$ on $W_{+}$given in Proposition 3. 5.

4. 3. Let $B$ be the subgroup of $G_{c}$ given by (1.14) and let $B_{o}$ $=\delta B \delta^{-1}$, where $\delta=\exp \sqrt{-1} e$. We set

$$
1=c+r_{0}{ }^{0},
$$

where $\mathfrak{c}$ and $\mathfrak{r}_{0}{ }^{0}$ are subalgebras of $\mathfrak{g}^{0}$ as in $\S 1$. Then $t$ satisfies $[\mathfrak{s}, \mathrm{t}]=0$.

Lemma 4. 3. Under the notations above, the Lie algebra bof $B_{o}$ coincides with $\mathcal{W}_{-}+\mathfrak{t}_{c}+\mathfrak{f}_{c}+\mathfrak{l}_{-}$.

Proof. By (1.4), (1.7) and (1.13), the Lie algebra $\mathfrak{b}$ of $B$ is decomposed in the following form:

$$
\mathfrak{b}=\bar{Q}\left(\mathfrak{r}^{-1}\right)+\left(\mathfrak{r}_{s}{ }^{0}\right)_{c}+\mathfrak{t}_{c}+\bar{Q}\left(\mathfrak{g}^{-1}\right)+\mathfrak{g}_{c}{ }^{0}+\mathfrak{F}_{c}{ }^{1}+\mathfrak{G}_{c}{ }^{2} .
$$

By Proposition 2.5, Ad $\delta\left(\bar{Q}\left(\mathfrak{g}^{-1}\right)+\mathfrak{G}_{c}{ }^{0}+\mathfrak{g}_{c}{ }^{1}+\mathfrak{G}_{c}{ }^{2}\right)=\mathfrak{f}_{c}+\mathfrak{p}_{-} . \quad$ Clearly $A d \delta\left(\bar{Q}\left(\mathfrak{r}^{-1}\right)+\mathrm{t}_{c}\right)=\bar{Q}\left(\mathfrak{r}^{-1}\right)+\mathrm{t}_{c} \subset \mathcal{W}_{-}+\mathrm{t}_{c}$. Let $x \in\left(\mathfrak{r}_{s}{ }^{0}\right)_{c}$. Then $A d \delta x$ $=x+\sqrt{-1} j_{0} x \in W_{-}$. Hence we have proved $A d \delta \mathfrak{b} \subset \mathfrak{b}_{0}$. By considering the equality $\operatorname{dim} \bar{Q}\left(\mathfrak{r}^{-1}\right)+\operatorname{dim}\left(\mathfrak{r}_{s}{ }^{0}\right)_{c}=\operatorname{dim} \mathcal{W}_{-}$, we get $A d \delta \mathfrak{b}=\mathfrak{b}_{o}$.

$$
\text { q.e.d. }
$$

Let $h_{o}$ be a holomorphic mapping: $\mathcal{U}_{c} \times \mathcal{W}_{+} \times \mathfrak{p}_{+} \rightarrow G_{c} / B_{o}$ given by

$$
h_{0}(u, w, z)=\pi_{0} \cdot \exp u \cdot \exp w \cdot \exp z,
$$


where $\pi_{o}$ denotes the projection of $G_{c}$ onto $G_{c} / B_{o}$. Note that $U_{c}$ $+W_{+}+\mathfrak{p}_{+}$is an abelian subalgebra of $\mathfrak{g}(D)_{c}$.

Lemma 4. 4. $h_{o}$ is a holomorphic diffeomorphism of $U_{c} \times W_{+}$ $\times \mathfrak{p}_{+}$onto an open set of $G_{c} / B_{o}$.

Proof. It is sufficient to prove that $h_{0}$ is injective. Now suppose that $a=\exp u \cdot \exp w \cdot \exp z \in B_{0}$. Let $E^{\prime}=E-E_{s}$. Since $\left[E^{\prime}, \mathfrak{g}\right]$ $=0, E^{\prime}$ is contained in $\mathrm{t}$. Therefore $A d a E^{\prime}=E^{\prime}+2 u+w \in \mathfrak{b}_{o}$, because $\mathrm{t} \subset \mathfrak{b}_{o}$. Hence by Lemma $4.3, u=w=0$. Recall that $Z=\frac{1}{2}\left(I_{s}+e-e^{*}\right)$ is in $\mathfrak{f}$ and hence in $\mathfrak{b}_{o}$. Therefore $A d$ a $Z=Z+[z, Z]=Z-\sqrt{-1} z \in \mathfrak{b}_{o}$. This implies $z=0$.

q.e.d.

Since $[[\mathcal{W}, \mathscr{W}], \mathscr{W}]=0$, we can see the following (cf. [1] or [10]):

(4.11) $\exp \left(w+w^{\prime}\right)=\exp w \cdot \exp w^{\prime} \cdot \exp \frac{1}{2}\left[w^{\prime}, w\right] \quad\left(w, w^{\prime} \in W_{c}\right)$.

For an element $w$ of $\mathscr{W}_{c}$, denote by $w_{+}$(resp. by $w_{-}$) its $\mathscr{W}_{+}$-(resp. $W_{--}$) component.

Lemma 4.5 (cf. [6]). Every $g \in G^{s}$ leaves $h_{o}\left(U_{c} \times \mathscr{W}_{+} \times \mathscr{S}\right)$ invariant and hence induces a holomorphic transformation $\widetilde{g}$ of $U_{c}$ $\times W_{+} \times \mathscr{S}$. Let $\widetilde{g}(u, w, z)=\left(u^{\prime}, w^{\prime}, z^{\prime}\right)$. Then

$$
\left\{\begin{array}{l}
z^{\prime}=g(z), \\
w^{\prime}=(g w)_{+}-z^{\prime}(g w)_{-}=g(g, z) w, \\
u^{\prime}=u-\frac{1}{2}\left[w^{\prime}, g w\right] .
\end{array}\right.
$$

Proof. By using (4.11), we obtain

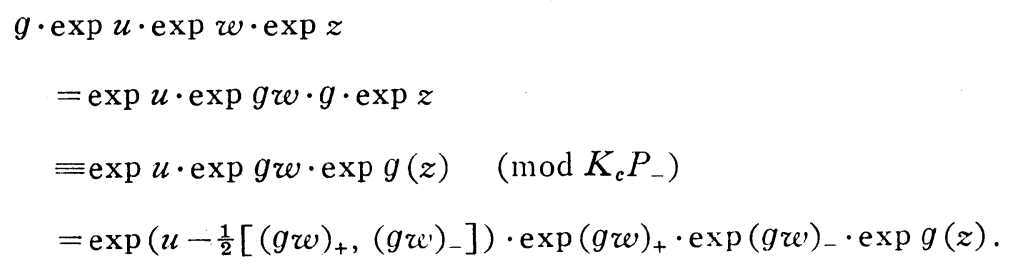

And 


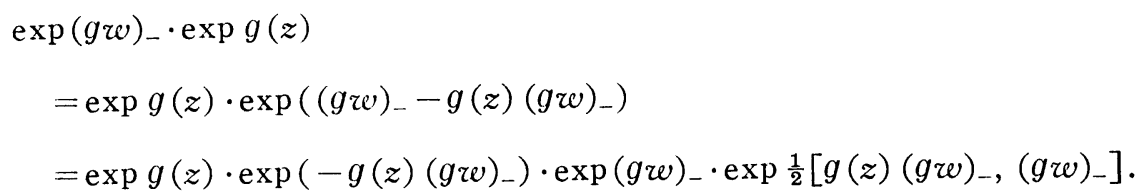

Therefore

$g \cdot \exp u \cdot \exp w \cdot \exp z$

$$
\begin{array}{r}
\equiv \exp \left(u-\frac{1}{2}\left[(g w)_{+},(g w)_{-}\right]+\frac{1}{2}\left[g(z)(g w)_{-},(g w)_{-}\right]\right) \\
\cdot \exp \left((g w)_{+}-g(z)(g w)_{-}\right) \cdot \exp g(z) \quad\left(\bmod B_{o}\right) .
\end{array}
$$

Hence $z^{\prime}=g(z), w^{\prime}=(g w)_{+}-g(z)(g w)_{-} \quad$ and $u^{\prime}=u-\frac{1}{2}\left[w^{\prime},(g w)_{-}\right]$ $=u-\frac{1}{2}\left[w^{\prime}, g_{w}\right]$. It remains to show $(g w)_{+}-g(z)(g w)_{-}=g(g, z) w$. We can write $g \cdot \exp z=\exp g(z) \cdot g(g, z) \cdot \exp \bar{z}_{1}\left(z_{1} \in \mathfrak{p}_{+}\right)$. Then $g w$ $=g \cdot \exp z w=g(g, z) w+g(g, z) \bar{z}_{1} w+g(z) g(g, z) \bar{z}_{1} w$. Therefore $(g w)_{+}$ $=g(g, z) w+g(z) g(g, z) \bar{z}_{1} w$ and $(g w)_{-}=g(g, z) \bar{z}_{1} w$. Hence we have $w^{\prime}=(g w)_{+}-g(z)(g w)_{-}=g(g, z) w$.

q.e.d.

Next we verify

Lemma 4.6 (cf. [6]). Let $g \in G^{s}$ and let $\widetilde{g}(u, w, z)=\left(u^{\prime}, w^{\prime}, z^{\prime}\right)$. Then $\operatorname{Im} u-\operatorname{Re} \mathcal{L}_{z}\left(w^{\prime}, w\right)=\operatorname{Im} u^{\prime}-\operatorname{Re} \mathcal{L}_{z^{\prime}}\left(w^{\prime}, w^{\prime}\right)$.

Proof. We first assume that $z=0$. By Lemma 4.5, $u^{\prime}=u-\frac{1}{2}$ $\times[g w, g w], w^{\prime}=g w$ and $z^{\prime}=g(0)$, here we put $g=g(g, 0)$. Therefore $\operatorname{Im} u^{\prime}-\operatorname{Re} \mathcal{L}_{z^{\prime}}\left(w^{\prime}, w^{\prime}\right)=\operatorname{Im} u-\frac{1}{2} \operatorname{Im}[g w, g w]-\operatorname{Re} \mathcal{L}_{z^{\prime}}(g w, g w)$. By direct calculations,

$$
\begin{aligned}
\operatorname{Re} \mathcal{L} & ,(g w, g w) \\
& =2 \sqrt{-1} \mathcal{H}\left(g_{w}, \mathcal{K}\left(z^{\prime}\right) \bar{g} \bar{w}\right)-2 \operatorname{Im} \mathscr{H}\left(g_{w}, \mathcal{K}\left(z^{\prime}\right) \bar{z}^{\prime} g_{w}\right) \\
& =2 \sqrt{-1} \mathcal{H}(w, \bar{w})-2 \operatorname{Im} \mathcal{A}\left(g_{w}, \bar{z}^{\prime} \mathcal{K}\left(z^{\prime}\right) g w\right) \\
& =\operatorname{Re} \mathcal{L}_{0}(w, w)-\frac{1}{2} \operatorname{Im}\left[g_{w}, \bar{z}^{\prime} \bar{g}_{w}\right],
\end{aligned}
$$

here we used the facts that $\mathcal{K}\left(z^{\prime}\right)=g \bar{g}^{-1}$ and $\bar{z}^{\prime} \overline{\mathcal{K}\left(z^{\prime}\right)}=\mathcal{K}\left(z^{\prime}\right) \bar{z}^{\prime}$ on $W_{+}$(cf. Proof of Lemma 4.5). Since $g=\exp z^{\prime} \cdot g \cdot \exp \bar{z}^{\prime \prime}\left(z^{\prime \prime} \in \mathfrak{p}_{+}\right)$, $g=\bar{g}=\exp \bar{z}^{\prime} \cdot \bar{g} \cdot \exp z^{\prime \prime}$. Hence $g w=\bar{g} w+\bar{z}^{\prime} \bar{g} w$ and $\left[g w, \bar{z}^{\prime} \bar{g} w\right]$ $=\left[g_{w}, g w\right]$. Combining these equalities, we get $\operatorname{Im} u^{\prime}-\operatorname{Re} \mathcal{L}_{z^{\prime}}\left(w^{\prime}\right.$, 
$\left.w^{\prime}\right)=\operatorname{Im} u-\operatorname{Re} \mathcal{L}_{0}(w, w)$. Since $\mathscr{S}$ is homogeneous, for any $z \in \mathscr{S}$ there exist $f \in G^{s}, u_{0} \in \mathcal{U}_{c}$ and $w_{0} \in \mathcal{W}_{+}$such that $\tilde{f}\left(u_{0}, w_{0}, 0\right)=(u, w, z)$. Hence $\widetilde{g f}\left(u_{0}, w_{0}, 0\right)=\left(u^{\prime}, w^{\prime}, z^{\prime}\right)$. It follows $\operatorname{Im} u^{\prime}-\operatorname{Re} \mathcal{L}_{z^{\prime}}\left(w^{\prime}, w^{\prime}\right)$ $=\operatorname{Im} u_{0}-\operatorname{Re} \mathcal{L}_{0}\left(w_{0}, w_{0}\right)=\operatorname{Im} u-\operatorname{Re} \mathcal{L}_{z}(w, w)$.

By Lemma 4.6, we know that each $\widetilde{g}\left(g \in G^{s}\right)$ leaves $D$ invariant. Moreover by Lemma 4.5 we know that $\widetilde{g}$ acts as a quasi-linear transformation in the sence of Pyatetski-Shapiro [5].

4. 4. Let $\tilde{\delta}$ be a holomorphic diffeomorphism of $G_{c} / B$ onto $G_{c} / B_{o}$ given by

$$
G_{c} / B \ni g B \underset{\delta}{\tilde{\delta}} g \delta^{-1} B_{o} \in G_{c} / B_{o}
$$

where $\delta=\exp \sqrt{-1} e$. Clearly $\tilde{\delta}$ is compatible with the action of $f$ $\in G$, i.e., $\tilde{\delta}(f p)=f \tilde{\delta}(p)\left(p \in G_{c} / B\right)$. We are now in a position to prove

Theorem 4.7. Let $\mathscr{D}$ be the Siegel domain of the third kind defined by (4.9) and let $h$ (resp. $h_{0}$ ) be the imbedding of $D$ (resp. of $D$ ) into $G_{c} / B$ (resp. into $G_{c} / B_{o}$ ) given by (1.15) (resp. by (4.10)). Then

$$
h_{o}(\mathscr{D})=\tilde{\delta} h(D)
$$

Proof. First we show that $h_{o}\left(\mathscr{D}_{o}\right)=\tilde{\delta} h\left(D_{o}\right)$. Let $u \in\left(\mathfrak{r}_{0}{ }^{-2}\right)_{c}, v$ $\in\left(\mathfrak{r}_{s}^{-2}\right)_{c}$ and $w \in \mathfrak{r}^{-1}$. Then

$$
\begin{aligned}
\tilde{\delta} h(u+v+w+\sqrt{-1} e) \\
\quad \equiv \exp u \cdot \exp v \cdot \exp Q(w) \\
\quad \equiv \exp u \cdot \exp v_{+} \cdot \exp v_{-} \cdot \exp \frac{1}{2}\left[v_{-}, v_{+}\right] \cdot \exp Q(w) \\
\quad \equiv \exp \left(u-\frac{1}{2}\left[v_{+}, v_{-}\right]\right) \cdot \exp \left(v_{+}+Q(w)\right) \quad\left(\bmod B_{o}\right)
\end{aligned}
$$

Therefore $\tilde{\delta} h(u+v+w+\sqrt{-1} c)=h_{o}\left(u^{\prime}, w^{\prime}, 0\right)$, where

$$
\left\{\begin{array}{l}
u^{\prime}=u-\frac{1}{2}\left[v_{+}, v_{-}\right], \\
w^{\prime}=v_{+}+Q(w) .
\end{array}\right.
$$


Since $v_{+}=\frac{1}{2}\left(v+\sqrt{-1}\left[e^{*}, v\right]\right)$ and $v_{-}=\frac{1}{2}\left(v-\sqrt{-1}\left[e^{*}, v\right]\right)$, we get $\frac{1}{2}\left[v_{+}, v_{-}\right]=\sqrt{-1} / 4\left[\left[e^{*}, v\right], v\right]$ and $\frac{1}{2}\left[v_{+}, \bar{v}_{+}\right]=\sqrt{-1} / 4\left[\left[e^{*}, v\right], \bar{v}\right]$. It follows

$$
\begin{aligned}
& \operatorname{Im} u^{\prime}-\operatorname{Re} \mathcal{L}_{0}\left(\mathfrak{w}^{\prime}, w^{\prime}\right) \\
& =\operatorname{Im} u-\frac{1}{4} \operatorname{Re}\left[\left[e^{*}, v\right], v\right]-\frac{\sqrt{-1}}{2}\left[v_{+}, \bar{v}_{+}\right]-\frac{\sqrt{-1}}{2}[Q(w), \bar{Q}(w)] \\
& =\operatorname{Im} u-\frac{1}{4} \operatorname{Re}\left[\left[e^{*}, v\right], v\right]+\frac{1}{4}\left[\left[e^{*}, v\right], \bar{v}\right]-\frac{1}{4}[[I, v], w] \\
& =\operatorname{Im} u-\frac{1}{4}[[I, w], w]-\frac{1}{2}\left[\left[\operatorname{Im} v, e^{*}\right], \operatorname{Im} v\right] .
\end{aligned}
$$

Hence by Corollary 3.3, $\left(u^{\prime}, w^{\prime}, 0\right) \in \mathscr{D}_{0}$ if and only if $u+v+w+\sqrt{-1} e$ $\in D_{o}$. Since for any $\left(u^{\prime}, w^{\prime}\right) \in U_{c} \times \mathcal{H}_{+}+$there exist unique $u \in\left({x_{0}}^{-2}\right)_{c}$ $\left(=\mathcal{U}_{c}\right), v \in\left(\mathfrak{r}_{s}{ }^{-2}\right)_{c}$ and $z \in \mathfrak{r}^{-1}$ satisfying (4.12), we get $h_{o}\left(\mathscr{D}_{o}\right)$ $=\tilde{\delta} h\left(D_{o}\right)$. Clearly $D=G^{s} D_{o}$ and $\mathscr{D}=\widetilde{G}^{s} \mathscr{D}_{o}$. Hence $h_{o}(\mathscr{D})=G^{s} h_{o}\left(\mathscr{D}_{o}\right)$ $=G^{s} \tilde{\delta} h\left(D_{o}\right)=\tilde{\delta} h\left(G^{s} D_{o}\right)=\tilde{\delta} h(D)$.

q.e.d.

4. 5. Since $D \cong(D)$ by Theorem 4. 7 , every $g \in \operatorname{Aut}(D)$ corresponds to a holomorphic transformation $\tilde{g}$ of $\mathscr{D}$. Then for $g \in G$ and $p \in \mathscr{D}$, the equality; $h_{o}(\widetilde{g}(p))=g h_{o}(p)$ holds, because the mappings $h$ and $\tilde{\delta}$ are compatible with the action of $G$.

Lemma 4. 7. Let $T$ be the connected subgroup of $G$ corresponding to the subalgebra $\mathrm{t}=\mathrm{c}+\mathrm{r}_{0}{ }^{0}$. Then for each $t \in T, \tilde{t}$ is a quasilinear transformation.

Proof. Let $(u, w, z) \in \mathscr{D}$. Then

$t \cdot \exp u \cdot \exp w \cdot \exp z \equiv \exp (A d t u) \cdot \exp (A d t w) \cdot \exp z \quad\left(\bmod B_{0}\right)$.

Since $A d t \circ j_{o}=j_{o} \circ A d t$, we know $A d t w \in \mathcal{W}_{+}$. Clearly $A d t u \in \mathcal{U}_{c}$. Therefore $\tilde{t}$ is a quasi-linear transformation of $\mathscr{D}$.

q.e.d.

Next we consider the action of the connected subgroup of $G$ corresponding to the subalgebra $U+W$. It is easy to see that this group coincides with $\exp \mathcal{U} \cdot \exp W$. 
Lemma 4.9. Let $f=\exp a \cdot \exp b \quad(a \in \mathcal{U}, b \in \mathcal{W})$, and let $\tilde{f}(u$, $w, z)=\left(u^{\prime}, w^{\prime}, z^{\prime}\right)$. Then

$$
\left\{\begin{array}{l}
z^{\prime}=z \\
w^{\prime}=w+b_{+}-z b_{-} \\
u^{\prime}=u+a+\frac{1}{2}[b, w]+\frac{1}{2}\left[b_{-}, b_{+}+w\right]-\frac{1}{2}\left[b_{-}, z b_{-}\right] .
\end{array}\right.
$$

In particular, $\tilde{f}$ is a parallel transformation in Pyatetski-Shapiro's sence $([5])$.

Proof. By using (4.11), one has

$f \cdot \exp u \cdot \exp w \cdot \exp z$

$$
\begin{aligned}
& =\exp (a+u) \cdot \exp (b+w) \cdot \exp \frac{1}{2}[b, w] \cdot \exp z \\
& =\exp \left(a+u+\frac{1}{2}[b, w]\right) \cdot \exp \left(b_{+}+w\right) \cdot \exp b_{-} \cdot \exp \frac{1}{2}\left[b_{-}, b_{+}+w\right] \cdot \exp z \\
& =\exp \left(a+u+\frac{1}{2}[b, w]+\frac{1}{2}\left[b_{-}, b_{+}+w\right]\right) \cdot \exp \left(b_{+}+w\right) \cdot \exp b_{-} \cdot \exp z
\end{aligned}
$$

Since $\exp b_{-} \cdot \exp z=\exp z \cdot \exp \left(b_{-}-z b_{-}\right) \equiv \exp z \cdot \exp \left(-z b_{-}\right) \cdot \exp \frac{1}{2}\left[z b_{-}\right.$, $\left.b_{-}\right]\left(\bmod B_{o}\right)$, we get

$$
\begin{aligned}
& f \cdot \exp u \cdot \exp w \cdot \exp z \\
& =\exp \left(a+u+\frac{1}{2}[b, w]+\frac{1}{2}\left[b_{-}, b_{+}+w\right]-\frac{1}{2}\left[b_{-}, z b_{-}\right]\right) \\
& \cdot \exp \left(b+w-z b_{-}\right) \cdot \exp z \quad\left(\bmod B_{o}\right) .
\end{aligned}
$$

4. 6. Define a Subgroup $G L(D)$ of $\operatorname{Aut}(D)$ by

$$
G L(D)=\left\{f \in G L\left(R_{c}+W\right) ; f(D)=D\right\} .
$$

Then $\operatorname{Aut}(D)=G \cdot G L(D)$ ([2] or [3]) and the Lie algebra of $G L(D)$ is $\mathrm{g}^{0}([2])$. By virtue of Lemma 4.5, Lemma 4.8 and Lemma 4.9, each element of $G$ corresponds to a quasi-linear transformation of $\mathscr{D}$. Therefore it remains to investigate the action of $G L(D)$ on $\mathscr{D}$.

For any $g \in G L(D)$, let us denote by $\tau(g)$ the isomorphism of $G_{c}$ given by

$$
\tau(g) a=A d(g) a A d(g)^{-1} \quad\left(a \in G_{c}\right) .
$$

Then $\tau(g) B=B$ and hence $\tau(g)$ induces an automorphism (denoted by 
the same letter $\tau(g))$ of $G_{c} / B$. From the definition of Tanaka's imbedding, we have

$$
h(g(p))=\tau(g) h(p) .
$$

We now put $\mathfrak{g}^{\prime}=A d g \mathfrak{g}$. Then $\mathfrak{g}^{\prime}$ is also a semi-simple graded subalgebra of $\mathfrak{g}(D)$ satisfying (1.3). Hence there exists $X$ in $\mathfrak{g}^{0}$ such that $A d(\exp X) \mathfrak{g}^{\prime}=\mathfrak{g}([4])$. Put $g^{\prime}=\exp X \cdot g$. Clearly $g^{\prime} \in G L(D)$ and $A d g^{\prime} \mathfrak{g}=\mathfrak{g}$. It follows $A d g^{\prime} V_{s}=V_{s}$ and hence there exists $Y_{1}$, $\cdots, X_{m} \in \mathfrak{g}^{0}$ such that $A d\left(\exp X_{1} \cdots \exp Y_{m}\right) A d g^{\prime} c=e$. Let $g^{\prime \prime}=\exp Y_{1}$ $\cdots \exp Y_{m} \cdot g^{\prime}$. Then $g^{\prime \prime}$ is an element of $G L(D)$ having the following properties :

$$
\text { a) } A d g^{\prime \prime} \mathfrak{g}^{\lambda}=\mathfrak{g}^{\lambda} \text { and } A d g^{\prime \prime} e=e \text {. }
$$

Moreover it is not difficult to show the equality;

b) $A d g^{\prime \prime} E_{s}=E_{s}$.

By using a), and b), we can see

c) $A d g^{\prime \prime} e^{*}=e^{*}$.

From a), b) and c), we know that the spaces $\mathfrak{p}_{+}, \mathfrak{p}_{-}, \mathcal{U}, \mathcal{W}_{+}$and $\mathcal{W}_{-}$ are stable under $A d g^{\prime \prime}$. Furthermore $\tau\left(g^{\prime \prime}\right) B_{o}=B_{o}$ and hence $\tau\left(g^{\prime \prime}\right)$ induces an autsmorphism of $G_{c} / B_{o}$, which is denoted by the same letter $\tau\left(g^{\prime \prime}\right)$. Obviously $\tau\left(g^{\prime \prime}\right) \circ \tilde{\delta}=\tilde{\delta} \circ \tau\left(g^{\prime \prime}\right)$. As a consequence we get for any $p \in D$,

$$
\tilde{\delta} h\left(g^{\prime \prime}(p)\right)=\tilde{\delta} \circ \tau\left(g^{\prime \prime}\right) \cdot h(p)=\tau\left(g^{\prime \prime}\right) \tilde{\delta} h(p) .
$$

Hence for any $(u, z, w) \in \mathscr{D}$,

$$
\begin{aligned}
h_{o}\left(\widetilde{g}^{\prime \prime}(u, z, w)\right) & =\pi_{o} \tau\left(g^{\prime \prime}\right)(\exp u \cdot \exp w \cdot \exp z) \\
& =\pi_{o} \exp \left(A d g^{\prime \prime} u\right) \cdot \exp \left(A d g^{\prime \prime}{ }^{\prime}\right) \cdot \exp \left(A d g^{\prime \prime} z\right)
\end{aligned}
$$

This equality leads us to say that $\widetilde{g}^{\prime \prime}$ (and therefore $\tilde{g}$ ) is a quasilinear transformation of $\mathscr{D}$. Thus we have proved the following.

Theorem 4. 10. In the realization of $D$ as $D$, each element of Aut(D) corresponds to a quasi-linear transformation and each clement of $\exp U \exp \mathscr{W}$ induces a parallel transformation of $\mathscr{D}$. 
Remark 2. It is not difficult to see that when the domain $D$ is homogeneous, our realization coincides with one given in Corollary 2, II-37 in Takeuchi [8].

\section{DEPARTMENT OF MATHEMATICS KYOTO UNIVERSITY}

\section{References}

[1] S. Kaneyuki, Homogeneous bounded domains and Siegel domains, Lecture Notes in Math. 241, Springer-Verlag, Berlin (1971).

[2] W. Kaup, Y. Matsushima and T. Ochiai, On the automorphisms and equivalences of generalized Siegel domains, Amer. J. Math., 92 (1970) 475-498.

[3] K. Nakajima, On Tanaka's imbeddings of Siegel domains, J. Math. Kyoto Univ., 14 (1974) 533-548.

[4] K. Nakajima, Symmetric spaces associated with Siegel domains, J. Math. Kyoto Univ., 15 (1975) 303-349.

[5] I. I. Pyatetski-Shapiro, Géométrie des domains classiques et théorie des functions automorphes, Dunod, Paris (1966).

[6] I. Satake, Realization of symmetric domains as Siegel domains of the third kind, Lecture Notes at Univ. of California (1972).

[7] I. Satake, Infinitesimal automorphisms of symmetric Siegel domains, (to apear).

[8] M. Takeuchi, Homogeneous Siegel domains, Pub. Study Group Geometry, No. 7, Tokyo (1973).

[9] N. Tanaka, On the equivalence problems associated with a certain class of homogeneous spaces, J. Math. Soc. Japan, 17 (1965) 103-139.

[10] N. Tanaka, On infinitesimal automorphisms of Siegel domains, J. Math. Soc. Japan, 22 (1970) 180-212.

[11] J. Vey, Sur la division des domains de Siegel, Ann. Sci. École Norm. Sup., 4 Série, 3 (1971) 479-506.

[12] È. B. Vinberg, Theory of homogeneous convex cones, Trans. Moscow Math. Soc., (1963) 340-403.

[13] J. Wolf and A. Korányi, Generalized Cayley transformations of bounded symmetric domains, Amer. J. Math. 87 (1965) 899-939. 